\title{
A comparative study of the method of Williamson Hall and the pattern of cadmium oxide nanoparticles for X-rays
}

\author{
Esraa Ahmed Mohammed \\ Phone; +964750 3489466 \\ Email: esraa.mohammed@uoz.edu.krd \\ Duhok, Iraq \\ University of Zakho \\ College of Basic Education / General Science Department
}

Article History: Received:11 January 2021; Accepted: 27 February 2021; Published online: 5 April 2021

\begin{abstract}
X-ray difraction (XRD) is an effective non-destructive instrument used in the determination and analysis of amorphous and crystalline materials. Three basic elements are the X-ray diffractometers: the X-ray tube, a retention of samples and an X-ray detector. In many industries such as diodes, transistors, detectors, solar and photovoltaic cells, cadmium oxide $\mathrm{CdO}$ nanoparticles are used. For this analysis, $\mathrm{CdO}$ nanoparticles are semiconductors (type) and band-gaps of $2.5 \mathrm{eV}$ and $1.98 \mathrm{eV}$ in direct and indirect bands using cadmium oxide. Several temperatures, effects and parameters such as texture coefficient (TC), dislocation density $(\mu)$, special area (SSA), and micro strain were measured and determined $(\mathrm{S})$. The peaks of the analysis were the extension of the nano structure, crystal size and grid pressure of the $\mathrm{CdO}$ and were measured using the Size Train Plot of WilliamsonHall (SSP). The composition of the particle is the cubic fluorite and spatial group Fm-3m (225). In the peaks resulting from the calcination process, strain enlargement was observed. Accordingly, the above procedure determined all physical parameters as a result of the diffraction effects.
\end{abstract}

\section{Review of literature}

Nanoparticles from hydroxyapatite (HA) have been prepared using a synthesis of the microwave in (2010) K. Venkateswarlu et al.[1]. Several techniques, including X-ray diffraction (XRD), Fourier infra-red transformation (FTIR), and electron transmission microscopy, have been used (TEM). The crystalline dimensions were determined by Scherrer and the W-H plot analysis of the X-ray peak expansion. The XRD results showed that the hydroxyapatite (HA) diffraction peaks were well crystallized with a hexagonal structure. The crystallite measurements measured with the $\mathrm{W}-\mathrm{H}$ plot methods were consistent with the TEM results.

The MOTEVALIZADEH et al.[2] are synthesizing the hydrothermal heating $\mathrm{ZnO}$ nanorods at 5, 10 and $15 \mathrm{hp}, 0$ $1 \mathrm{~m} \mathrm{ZnCl} 2$ at $10 \mathrm{pH}$. XRD trends have shown that hydrothermal treatment times will influence the preferred nanorod orientation. In Williamson-Hall and Halder-Wagner approaches, the crystallite dimensions and microstrains were analyzed. These findings demonstrate $\mathrm{ZnO}$ nanorod defects. Crystallite and grid defects are however reduced by increasing the duration of hydrothermal therapy.

Tungsten carbide nanoparticles were synthesized with a chemical method in (2013) Gourav Singla et al.[3]. Cristallite size and gate pressure were calculated in the Williamson-Hall $(\mathrm{W}-\mathrm{H})$ sample using X-ray diffraction (XRD) analysis. For all peaks of XRD reflexion, physical parameters including voltage, stress and energy density values were measured accurately. The particle size of tungsten carbide derived from the TEM pattern is around $38 \mathrm{~nm}$. The TEM findings are very consistent with the results of the Williamson-Hall $(\mathrm{W}-\mathrm{H})$.

In (2013): Baruah Bhaskarjyoti et al.

The Chemical Bath Deposition Process (CBD) has been deposited in nanocrystalline PbS films. The structural parameters of $\mathrm{PbS}$ films were analyzed using Williamson Hall and Modified Williamson Hall's X-ray line analysis process. Film stress varies between 7,4x10-4 and 2,82 x 10-3 and dislocation ranges from $\sim 1015$ to $€ 1016 \mathrm{sqm}$.

In (2013) Y. T. Prabhu et al.[5], the fe-doped ZnO procedure is prepared at various doping levels of 2 to 10 percent by means of the supported combustion surfactant synthesis. The synthesized samples were described by the $\mathrm{x}$-ray particles and TEM diffraction analyser. The decrease in crystallite sizes was observed with increased doped nanoparticles of Fe-doped ZnO. Fe doped Zno-NPs analyzed crystallite and grid strain using WilliamsonHall's stress inspection and scale patch $(\mathrm{W}-\mathrm{H})$. The XRD summit of all samples was used to describe pressure, stress and energy density parameters. 
UDM, Uniform Model of Stress Deformation (USDM), Uniform Model for Deformation of Energy Density (UDEDM) (SSP). The results showed a decrease in the stress value, but the doping concentration increased with the particle size. The TEM results were W-H and SSP compatible.

In (2015), S.N. Anitha and I. Jayakumari[6] were developed and characterized with the XRD and SEM analysis, using thermochemical solid-state analysis for nano-crystalline superconductive pottery Fe2Mn2Ni0.5Zn1.5O9. The Williamson Hall method calculated the crystalline dimensions and grind pressure of the peak expansion with the X-ray instrumental peak expansion analysis. The findings of an extension study using the Scherrer map, the W-H-plot method and the Size-Strain plot method are strongly correlated.

In (2015) the BSFTO nanoparticles with solid state-of-the-art technique were characterized by X-ray diffraction (XRD) and SEM analyzes in the Reenu Jacob and Jayakumari Isac[7]. BSFTO crystallite growth is investigated by X-ray peak expansion methods, such as Scherrer and Williamson Hall Plot Analysis (W-H). Results showed that the medium size measured by various methods of BSFTO crystallite is very interlinked. The SEM findings also coincide well with the W-H and SSP process results.

Nanoparticles (NPs) of Zn1-xCuxFe2O4(ZCFO) were synthesized in Dinesh Kumar et al. [9]. (2015). The ZCFO NPs' crystalline growth was studied by the maximum expansion of the XRD pattern. The plot method WilliamsonHall (W-H) was used to investigate the size and grid strain of ZCFO nPs for full expansion. At Cu2+ ion concentrations the specified value of crystallite size increases and minimized peak expansion.

The nanoparticles Pure and $\mathrm{La}-\mathrm{Zr}$ Co-doped $\mathrm{ZnO}$ were made by sol-gel techniques in Hossein Mahmoudi Chenari et al. (2016). X-ray diffraction (XRD) and measurement of electron transmission microscopy (TEM). The size and stress of the extension line were evaluated using the Williamson-Hall method and the Beach Plot method (SSP). The crystallite sizes of different models are approximately the same, with the average particle size obtained from the TEM results matching the USDM model's results well.

Silver silicone nitride was installed on Ti6Al4V alloy in (2017), Umi Zalilah and R Mahmoodians[9] using magnetic sputter technics for physical vapor deposition. AgSiN is characterized by field emission spectroscopy, dispersive electron spectroscopy (EDS) and X-ray diffraction in the morphology, compound and structure of deposited and thermal treatment coats (XRD). In the meantime, an important Williamson-Hall plot method was used to calculate the crystallite size and strain. Compared to a thermal sheet, the structure displayed a larger crystallite covering after therapy and a greater stress than a layer deposited.

Measuring the distance of the height of diffraction (2017) R. Rai Triloki et al.[11] looked at KBr thin film's microcrystalline properties. Hall of Williamson,

Size-Strain Single Line approaches Plot and Voigt. The coefficient of texture and dislocation density were calculated at each peak of diffraction.

For other related physical parameters, for example stress, young modulus and energy density, the WilliamsonHall approach to consistent stress deformation and deformation density is also evaluated. The findings are opposed to other approaches. Voigt's average value is greater than the Williamson-Hall and Size-Stress Chart values.

CdS nanoparticles have been synthesized with the R G Solanki1 and P Rajaram et al.[12] nonaqueous Solvo thermal method, while CdS nanoparticles have been measured by X-ray crystallite profile analysis. For size measurements, the Williamson-Hall (W-H) process, the Scherrer formula and SSP have been used. The results show that the size of crystallite, W-H maps, SSP and TEM image particles determined by Scherrers formula are approximately the same.

Zinc oxide nanoparticles $(\mathrm{ZnO})$ were synthesized under various reaction periods at $160 \mathrm{oC}$ in H.S. Wally et al. (2018)[13]. The XRD design lines are used as a simple and effective way in which grid stress and crystallite scale can be estimated. Crystallite scale and X-ray strain patterns have been analyzed using Scherrer and WilliamsonHall therapies. The XRD model verified the existence of crystal, hexagonal $\mathrm{ZnO}$ nanoparticles with different reaction times and an average crystallite size of 30 to $36 \mathrm{~nm}$.Nanocrystalline Ti alloy cry milling powder was developed in (2018) M. OH and H.K. YU, et al.[15]. The development of grain and crystal grid stress was evaluated using the Scherrer, Williamson-Hall (W-H) and Size-Plot (SSP) techniques. Additional physical parameters were measured, including stress and strain. The average crystallite size and strain of the XRD door is very similar to the results of electron microscopic transmission (TEM).

The feed-doped TiO2 nanoparticles have been prepared by the updated co-precipitation method in (2018) L. B. Patel et al.[15]. The Scherer method and the Williamson Hall method were used for the study of the X-ray peak 
enlargement to measure crystalline size and grid strain. The results for crystalline measurements and grid strains determined by both methods were roughly equivalent.

In (2018) MgO nanoparticles were synthesized in this study by Wayan Sutapa et al.[16] using the sol-gel method. Various techniques were employed to quantify crystallite quantities, including an XRD MgO nanoparticles, in other versions, UDM, UDSM and UDEDM, by means of the Scherrer equation, to quantity stress, energy density and stress. TC hkl was calculated The result shows that $\mathrm{MgO}$ nanoparticles are distributed in delivery particles in crystallite sizes below $10 \mathrm{~nm}$. The TC hkl measuring results show the favorite orientation of the MGO nanoparticles in the sample).(111)

Saba Fayazzadeh and Mehdi Khodaei[17] cobalt-ferrite nanoparticles were synthesized via the self-combustion process for citrate-nitrate solgel in (2019). The sample is synthesized with conventional automated combustion and the standard salt process is reduced to $11 \mathrm{~nm}$. Crystallite is $38.5 \mathrm{~nm}$. The Williamson-Hall equation indicates that the structure of the grid has been stressed by salt.

In (2019) I.P. Yaremi and al.[18], Zn-doped CoFe2O4 was synthesized on a chemical co-precipitation route. The crystallite size changes from 46 to $77 \mathrm{~nm}$ with $\mathrm{Zn}$ content. In the study of crystallite sizes, the Scherrer, Williamson-Hall (W-H) and the plot size method were used. The TEM results were well in keeping with the SSP system results.

Cadmium selenide (CdSe) nanoparticles were prepared in (2020) Debojyoti Nath et al. [19] using a chemistry process. The study on X-rays (XRD) explains the crystalline structure of the nano-particles of the CdSe composing the combination from the cubic zinc grid, whereas (ME) electro microscopy (AFM). The crystal size has been determined using the Williamson-Hall $(\mathrm{W}-\mathrm{H})$ and Halder-Wagner $(\mathrm{H}-\mathrm{W})$ methods. The findings of the SizeStrain Plot (SSP) and Halder-Wagner (H-W) methods fit the medium sized TEM, AFM and SEM samples very well.

\subsection{Planning}

In this section the maximum expansion of the Williamson-hall CDO nano-structure and other methods such as Debye Scherrrer, modified Scherrrer and an integrated distance, frequently applied with different calcinating temperatures and effects on size and strain, are examined (S).

\subsection{Diffraction of X-ray}

X-ray difraction (XRD) is an effective non-destructive instrument used in the determination and analysis of amorphous and crystalline materials. In 1914, the interference with radiation reflections was found to be a cause of X-ray diffraction, by Sir W.H. Bragg and his son W.L. Bragg[20]. Each plane reflects just a little radiation. On one level, each atom becomes the source of a dispersed X-ray, and the dispersed X-ray typically combines with any atom in a crystal as it fits aloofly on top of each other. In the parallel planes of atoms in the crystal, in complete crystal, waves of occurrence are expressed with radiological disorders without energy loss. Bragg reflects partial reflection and intrusion. The words diffraction and reflection are Bragg. Bragg has therefore established a basic mathematical relationship that is a precondition for Bragg's thinking[21]. Under the Bragg rules.

$$
\mathrm{n} \lambda=2 \mathrm{~d}_{\mathrm{hk} 1} \sin \theta
$$

Where $\mathrm{d}$ : distance between planes, $\beta$ : beam longitude, os: incidence beam angle, $\mathrm{n}$ : divisions number of order. The Bragg law showed a sequence of aircraft similar to hkl-military indicators that distinguish between dhkl wavelength $\mu$ and xrays of planes. As this beam hits the aircraft at an angle, the angle is the same as in Figure 2.1.

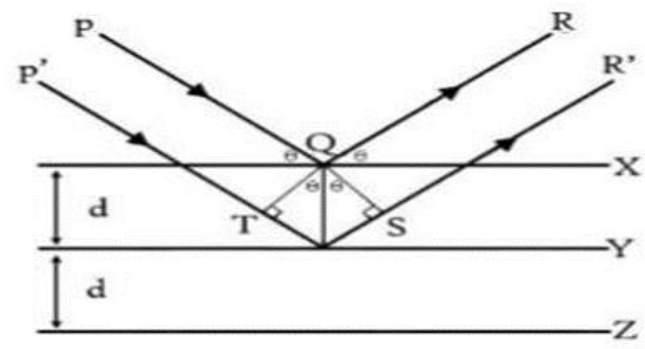




\subsection{Diffractometer X-ray}

Figure 2.2 has three basic components: X-ray tube, sample holder and X-ray detector. Radiation is produced by filament heating, using voltage to accelerate the electrons in the objective, to develop electrons in a cathode ray tube and by bombarding the target material with electrons. If electrons are strong enough to dislodge electrons from the internal shell into the target material, typical x-ray spectra are produced. These spectrums are composed of many elements, $\mathrm{K} \alpha$ and $\mathrm{K} \beta$ being the most common.

$\mathrm{K} \alpha$ is made up of $\mathrm{K} \alpha 1$ segment and $\mathrm{K} \alpha 2$ section. The wavelength of the $\mathrm{K} \alpha 1$ and the intensity of the $\mathrm{K} \alpha 2$ are two times shorter. Typical are the unique wavelengths of the target material $(\mathrm{Cu}, \mathrm{Fe}, \mathrm{Mo}, \mathrm{Cr})$. It is necessary to filter the monochromatic X-rays needed to diffract by foils or by crystal monochrometers. The wavelength of K $\alpha 1$ and $\mathrm{K} \alpha 2$ is enough for the use of the two weighted average. Copper is the most common radiation object for singlecrystal diffraction $=1.5418 \mathrm{~A} 0$. These X-rays are sent and collimated into the sample. During the sample and detector rotation, the strength of the $\mathrm{X}$-rays is reported. When the radiation incident geometry is met.

Law of Bragg, positive intervention and seriousness. It is captured and analyzed through a detector and then translated into a count rate, which is then produced into an appliance like a printer or a computer monitor. The geometry of the X-ray diffractometer indicates that the sample travels towards the collimated X-ray beam in a rotational angle and the X-ray is located on the arm for absorbing and turning the diffracted $\mathrm{x}$-rays at a $2^{\circ} \mathrm{C}$ angle.

In order to compare the pattern with the pdf file of the international data center and measured crystallite, the position of the pinnacle in the XRD pattern was analyzed by the Scherrer Formula powder method[23].

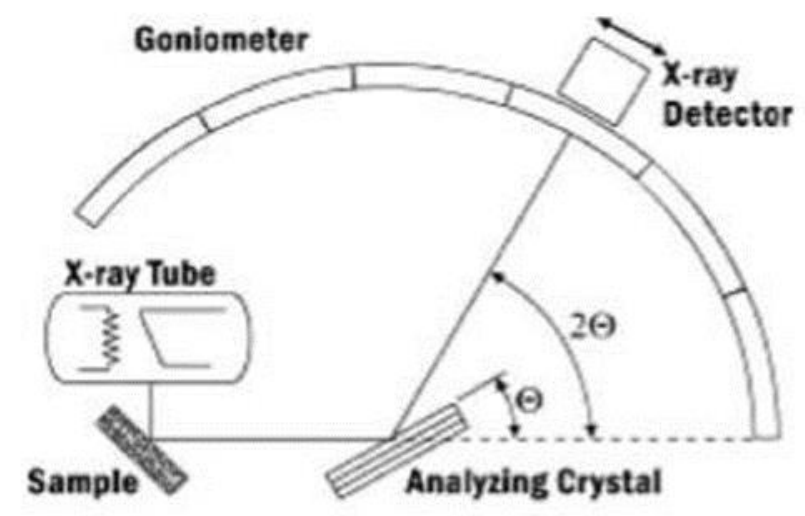

\subsection{Size and strain of crystallites}

\subsubsection{Developments}

Crystallite size: associated with the field size that sometimes diffracts and has not the particle size due to particle size and the X rays are more prone to treatment] 24 [Lattice strain: assignment of the constants determined by crystal defects, such as the dislocation of the grid[25].

\subsection{Strain types}

Three strains affect the top shape as shown in figure 2,5)

1: No tension, crystallites seem to be untrained.

2 :Uniform stress; the crystallites have a uniform tensile and their distance would be bigger.

3: The crystallites of this kind were bent; but due to a strain on the top, the distance of the plane was exceeded $\mathrm{d}$ and the aircraft was spaced below $\mathrm{d}$ by compressions[26]. 


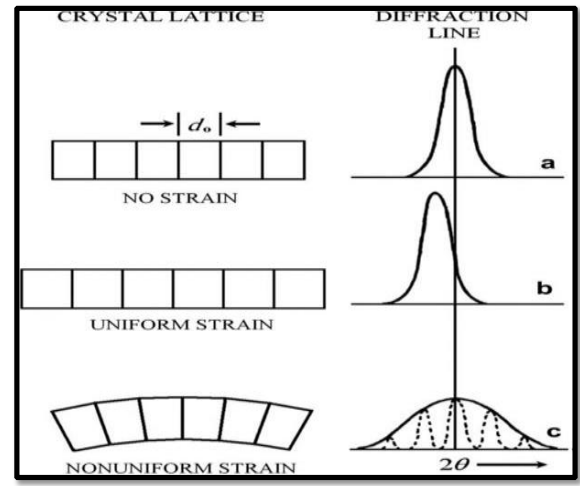

Figure (2.5): a- no strain, b- uniform strain, c-non uniform strain [27].

\subsection{The oxide of cadmium}

The (typical) semi-conductor and belt laps of 2,5 eV and 1,98 eV are used in direct and indirect cadmium oxide CdO nanoparticles for the study[28]. It offers a wide range of applications in the field of photocatalyst, gas sensors, transparent and solar cell applications, diodes, transistors, detectors, solar and photovoltaic cells. It is also common in drugs, antimicrobials, optics and environmental rehabilitation[29]. It is also widely used. A FCC with high intensity polycrystalline peaks of $111,200,220,311$, as shown in the XRD (2.1) model, was composed of the cadmium oxide Nanoparticle XRD model[30].

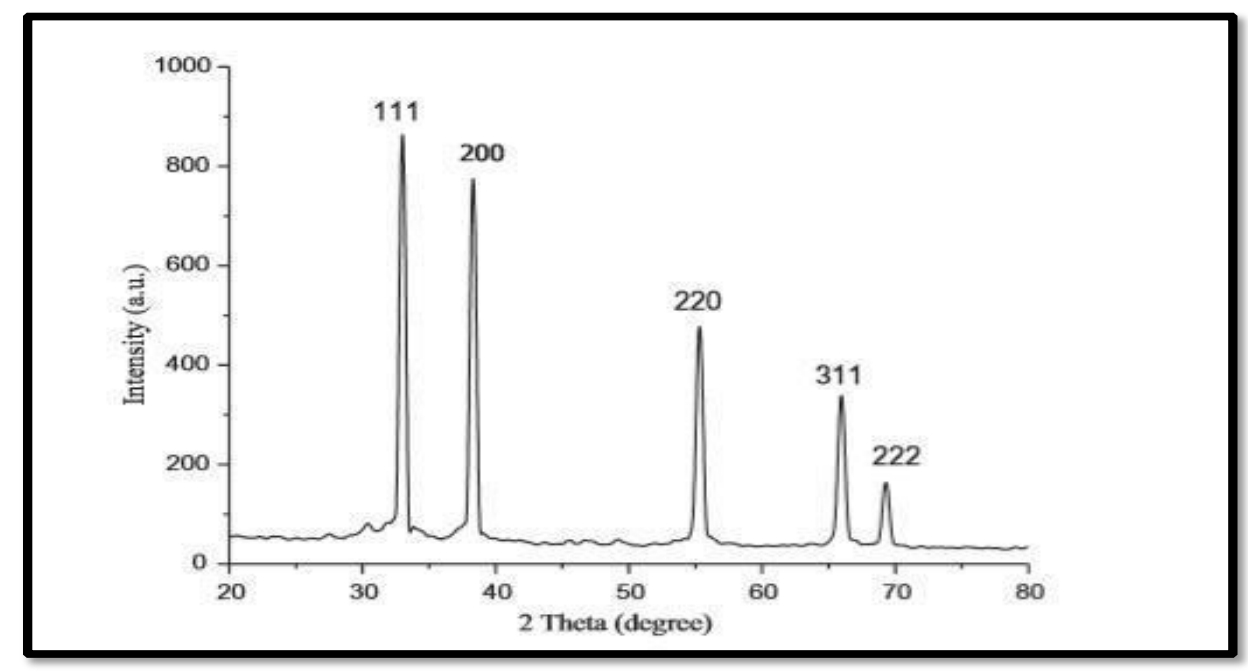

Figure( 2.1): XRD of CdO nanoparticles [30].

\subsection{Parameters of the Lattice}

\subsubsection{Density of dislocation}

The dislocation density is the length of lines per crystal volume[31]. Dislocation within a crystal structure is a crystallograph deformation or irregularity. Dislocation can affect the material's properties. It is a kind of topological imperfection, scientifically speaking. It increases with plastic deformation; as a dislocating instrument the material must be initiated. Dislocation involves three standardized nucleation processes, grain boundary initiation and grid and surface interface $[32,33]$. The density of the sample dislocation (ć) was calculated by expressions.

$$
\delta=\overline{\mathrm{D}^{2}}
$$




\subsubsection{Specific area of surface (SSA)}

Material property is a fixed surface area (SSA). This is a scientific value that can be used to assess the material's form and properties. It is particularly necessary when adsorption, heterogeneous catalysis and surface response occur.

$S=\frac{6 * 10^{3}}{D \rho}$

$\mathrm{S}:$ the specific surface area $\mathrm{D}$ is the crystallite size $\rho$ : the density $8.15 \mathrm{~g} / \mathrm{cm}^{3}$ for $\mathrm{CdO}[34,35]$.

\subsubsection{Coefficient of texture}

A number of texture coefficients listed in: $[36,37]$ provide quantitative details concerning the preferential orientation of crystallites.

$\mathrm{TC}_{(\mathrm{h} \mathrm{k} \mathrm{l})}=\frac{\frac{\mathrm{I}_{(\mathrm{h} \mathrm{k} \mathrm{l})}}{\mathrm{Io}_{(\mathrm{h} \mathrm{k} \mathrm{l})}}}{\frac{1}{n} \sum_{n} \frac{\mathrm{I}_{(\mathrm{h} \mathrm{k} \mathrm{l})}}{\mathrm{Io}_{(\mathrm{h} \mathrm{k} \mathrm{l})}}}$

$\mathrm{TC}_{(\mathrm{hkl})}$ : is the measured texture coefficient. $\mathrm{I}_{(\mathrm{hkl})}$ : is the measured intensity of the XRD peaks.

$\mathrm{n}:$ is the measured number of diffraction peaks.

$\mathrm{I}_{\mathrm{o}(\mathrm{h} \mathrm{kl})}$ : is the measured intensity of the reference in the XRD [38].

\subsubsection{Micro Strains (S)}

During forming of thin foil, the micro strains are caused by expansion or compression in the grid to produce a deviation in the grid constant. The expansion of the strain is thus induced by the different displacements of the atoms in respect of their point of reference[39, 40]. This strain can be tested using the formula [41].

$<S>=\frac{\beta \cos \theta}{4}$

$\beta$ : FWHM (radian) $\theta$ : Bragg diffraction angle of the XRD peak (degree)

\subsection{Different calcination temperature X-ray diffraction pattern}

In Figure $2.7(400 \mathrm{OC}, 600 \mathrm{OC}$ and $800 \mathrm{OC})$ for a nanopart of $\mathrm{CdO}$, the effect of various temperatures in the xRD pattern was detected; the calcination of specimens, and x-ray diffraction spikes were approved for planes (111), (220) and (311). [42]

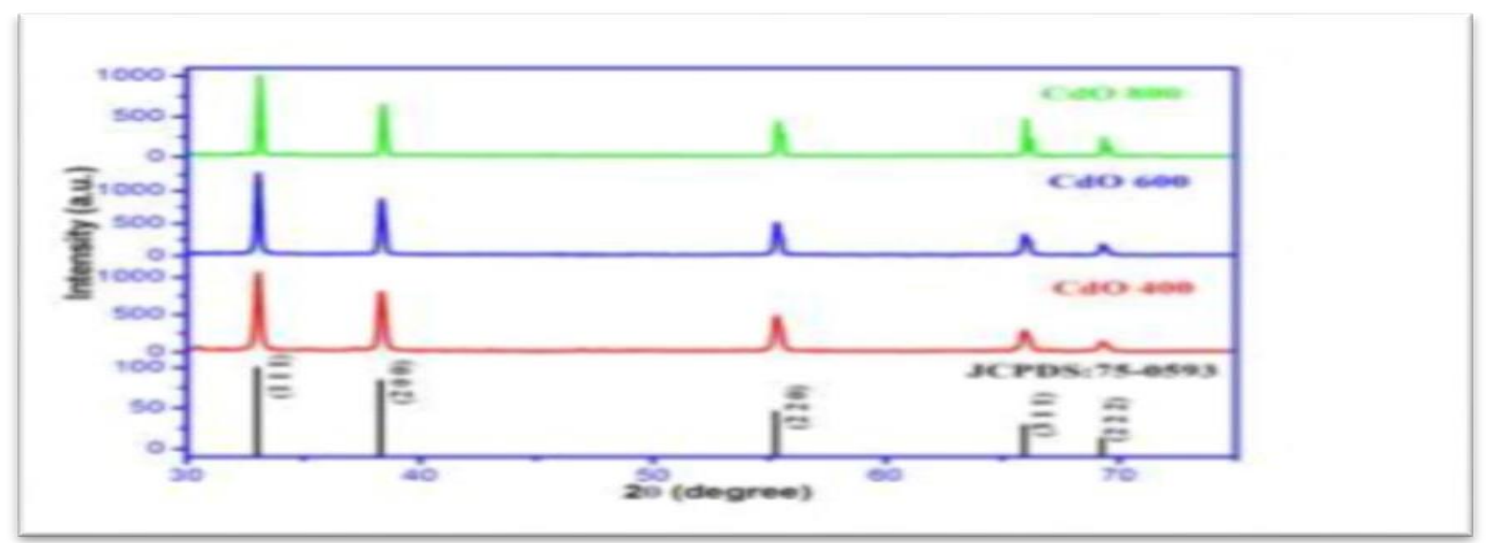

Fig. 2 XRD spectra of CdO nanoparticles calcined at 400, 600 and 


\section{Concluding}

The effect on the microstructure of the $\mathrm{CdO}$ nanopart cadmium oxide has been allocated to different calcination temperatures (400 OC, $600 \mathrm{OC}$ and $800 \mathrm{OC}$ ). The result was a micro-cristalline cubic design with a milling index (111), (200), (220) and (311). The expansion of strains as a reference grid due to the variable atomic changes resulted from the micro strains produced by thin films during the growth and the extension or compression of the grid that caused the a-gage constant to change.

\section{References}

1. K. Venkateswarlu, A. Chandra Bose, and N. Rameshbabu, "X-ray peak broadening studies of nanocrystalline hydroxyapatite by Williamson-Hall analysis," Physica B: Condensed Matter, vol. 405, pp. 4256-4261, 2010/10/15/2010.

2. L. Motevalizadeh, Z. Heidary, and M. E. Abrishami, "Facile template-free hydrothermal synthesis and microstrain measurement of $\mathrm{ZnO}$ nanorods," Bulletin of Materials Science, vol. 37, pp. 397-405, 2014/05/01 2014.

3. G. Singla, K. Singh, and O. P. Pandey, "Williamson-Hall study on synthesized nanocrystalline tungsten carbide (WC)," Applied Physics A, vol. 113, pp. 237-242, 2013/10/01 2013.

4. L. S. Bhaskarjyoti Baruah, Mothura Nath Bora, "X-Ray Line Profile Analysis of Chemically Deposited Nanostructured PBS Films," IOSR Journal of Applied Physics, vol. 5, pp. 07-13, 01/01 2013.

5. Y. Prabhu, K. V. Rao, V. S. S. Kumar, and B. S. Kumari, "X-ray analysis of Fe doped ZnO nanoparticles by Williamson-Hall and size-strain plot methods," Int. J. Eng. Adv. Technol., vol. 2, pp. 268-274, 2013.

6. S. Anitha and I. Jayakumari, "Synthesis and Analysis of Nanocrystalline Fe 2 Mn 2 Ni 0.5 Zn $1.5 \mathrm{O} 9$ at Different Treating Temperatures," Journal of Nanoscience and Technology, pp. 26-31, 2015.

7. H. G. N. Reenu Jacob, Jayakumari Isac, "Structural and Morphological Studies of Nanocrystalline Ceramic BaSr0.9Fe0.1TiO4," International Letters of Chemistry, Physics and Astronomy, vol. 41, pp. 100--117, 2015.

8. D. Kumar, A. Kumar, R. Prakash, and A. K. Singh, "X-ray diffraction analysis of Cu2+ doped Zn1xCuxFe2O4 spinel nanoparticles using Williamson-Hall plot method," AIP Conference Proceedings, vol. 2142, p. 070018, 2019/08/29 2019.

9. H. M. Chenari, H. F. Moafi, and O. Rezaee, "A study on the microstructural parameters of $\mathrm{Zn}(1-\mathrm{x})$ LaxZrxO nanopowders by X-ray line broadening analysis," Materials Research, vol. 19, pp. 548-554, 2016.

10. U. Zalilah and R. Mahmoodian, "Comparative study on microstructure, crystallite size and lattice strain of as-deposited and thermal treatment silver silicon nitride coating on Ti6Al4V alloy," in IOP Conference Series: Materials Science and Engineering, 2017, p. 012074.

11. R. Rai, T. Triloki, and B. K. Singh, "X-ray diffraction line profile analysis of KBr thin films," Applied Physics A, vol. 122, p. 774, 2016/07/30 2016.

12. R. G. Solanki and P. Rajaram, "Structural, optical and morphological properties of CdS nanoparticles synthesized using hydrazine hydrate as a complexing agent," Nano-Structures \& Nano-Objects, vol. 12, pp. 157-165, 2017/10/01/ 2017.

13. H. Wasly, M. Abd El-Sadek, and M. Henini, "Influence of reaction time and synthesis temperature on the physical properties of $\mathrm{ZnO}$ nanoparticles synthesized by the hydrothermal method," Applied Physics A, vol. 124, p. 76, 2018.

14. H. K. Y. M. OH, J.-H. LEE, M.C. OH, S.-H. JUNG, B. AHN, "QUANTITATIVE ANALYSIS OF CRYOMILLED NANOCRYSTALLINE Ti-6Al-4V ALLOY POWDER BY X-RAY DIFFRACTION," Arch. Metall. Mater, vol. 63, pp. 1439-1442, 2018.

15. L. Patle, P. Labhane, V. Huse, and A. Chaudhari, "Structural analysis of $\mathrm{Cu}$ doped TiO2 nanoparticles using Williamson-Hall method," International Journal of Scientific Research in Science, Engineering and T echnology, vol. 1, pp. 66-70, 2015.

16. I. W. Sutapa, A. W. Wahab, P. Taba, and N. La Nafie, "Synthesis and structural profile analysis of the $\mathrm{MgO}$ nanoparticles produced through the sol-gel method followed by annealing process," Oriental Journal of Chemistry, vol. 34, p. 1016, 2018.

17. M. Khodaei and S. Fayazzadeh, "Magnetic properties of $\mathrm{CoFe} 2 \mathrm{O} 4$ nanoparticle synthesized by saltassisted sol-gel auto-combustion method," Materials Research Express, vol. 6, p. 086115, 2019/06/19 2019.

18. I. Yaremiy, V. Bushkova, N. Bushkov, and S. Yaremiy, "X-ray Analysis of NiCrxFe2-xO4 Nanoparticles Using Debye-Sherrer, Williamson-Hall and Size-strain Plot Methods," Journal of nano-and electronic physics, pp. 04020-1-04020-8, 2019. 
19. D. Nath, F. Singh, and R. Das, "X-ray diffraction analysis by Williamson-Hall, Halder-Wagner and sizestrain plot methods of CdSe nanoparticles- a comparative study," Materials Chemistry and Physics, vol. 239, p. 122021, 2020/01/01/ 2020.

20. W. H. Bragg, "X-Rays and Crystalline Structure," Science, vol. 40, pp. 795-802, 1914.

21. N. Fourikis, "5 - Beamformers," in Advanced Array Systems, Applications and RF Technologies, N. Fourikis, Ed., ed London: Academic Press, 2000, pp. 325-353.

22. D. Mandal, Ultra-thin films of a ferroelectric copolymer: $P(V D F-T r F E), 2012$.

23. B. Ingham and M. F. Toney, "1 - X-ray diffraction for characterizing metallic films," in Metallic Films for Electronic, Optical and Magnetic Applications, K. Barmak and K. Coffey, Eds., ed: Woodhead Publishing, 2014, pp. 3-38.

24. W.-N. Wang, W. Widiyastuti, T. Ogi, I. W. Lenggoro, and K. Okuyama, "Correlations between Crystallite/Particle Size and Photoluminescence Properties of Submicrometer Phosphors," Chemistry of Materials, vol. 19, pp. 1723-1730, 2007/04/01 2007.

25. S. K. Mishra, H. Roy, A. K. Lohar, S. K. Samanta, S. Tiwari, and K. Dutta, "A comparative assessment of crystallite size and lattice strain in differently cast A356 aluminium alloy," IOP Conference Series: Materials Science and Engineering, vol. 75, p. 012001, 2015.

26. G. B. Greenough, "Strain Measurement by X-ray Diffraction Methods," Aeronautical Quarterly, vol. 1, pp. 211-224, 2016.

27. B. Nasiri-Tabrizi, "Thermal treatment effect on structural features of mechano-synthesized fluorapatitetitania nanocomposite: A comparative study," Journal of Advanced Ceramics, vol. 3, pp. 31-42, 03/01 2014.

28. P. A. Radi, A. G. Brito-Madurro, J. M. Madurro, and N. O. Dantas, "Characterization and properties of $\mathrm{CdO}$ nanocrystals incorporated in polyacrylamide," Brazilian Journal of Physics, vol. 36, pp. 412-414, 2006.

29. R. P. Lefojane, B. T. Sone, N. Matinise, K. Saleh, P. Direko, P. Mfengwana, et al., "CdO/CdCO3 nanocomposite physical properties and cytotoxicity against selected breast cancer cell lines," Scientific Reports, vol. 11, p. 30, 2021/01/08 2021.

30. T. S. Aldeen, H. E. A. Mohamed, and M. Maaza, "Bio-inspired Single Phase Monteponite CdO Nanoparticles via Natural Extract of Phoenix roebelenii Palm Leaves," Journal of Inorganic and Organometallic Polymers and Materials, vol. 30, pp. 4691-4701, 2020/11/01 2020.

31. A. Arsenlis, D. M. Parks, R. Becker, and V. V. Bulatov, "On the evolution of crystallographic dislocation density in non-homogeneously deforming crystals," Journal of the Mechanics and Physics of Solids, vol. 52, pp. 1213-1246, 2004/06/01/ 2004.

32. P. R. Rios, F. Siciliano Jr, H. R. Z. Sandim, R. L. Plaut, and A. F. Padilha, "Nucleation and growth during recrystallization," Materials Research, vol. 8, pp. 225-238, 2005.

33. A. L. Greer, "Overview: Application of heterogeneous nucleation in grain-refining of metals," The Journal of Chemical Physics, vol. 145, p. 211704, 2016/12/07 2016.

34. C. Amador and L. Martin de Juan, "Chapter 19 - Strategies for Structured Particulate Systems Design," in Computer Aided Chemical Engineering. vol. 39, M. Martín, M. R. Eden, and N. G. Chemmangattuvalappil, Eds., ed: Elsevier, 2016, pp. 509-579.

35. S. Senthil, S. Srinivasan, T. Thangeeswari, and V. Ratchagar, "Enrichment of optical, magnetic and photocatalytic properties in PVP capped $\mathrm{CdO} / \mathrm{SnO} 2$ nanocomposites synthesized by microwave irradiation method," Journal of Materials Science: Materials in Electronics, vol. 30, pp. 19841-19853, 2019/11/01 2019.

36. M. H. Babu, B. C. Dev, and J. Podder, "Texture coefficient and band gap tailoring of Fe-doped $\mathrm{SnO} 2$ nanoparticles via thermal spray pyrolysis," Rare Metals, 2019/07/11 2019.

37. K. Gurumurugan, D. Mangalaraj, and S. K. Narayandass, "Structural characterization of cadmium oxide thin films deposited by spray pyrolysis," Journal of Crystal Growth, vol. 147, pp. 355-360, 1995/02/01/ 1995.

38. Q. Luo and S. Yang, "Uncertainty of the X-ray Diffraction (XRD) $\sin 2 \psi$ Technique in Measuring Residual Stresses of Physical Vapor Deposition (PVD) Hard Coatings," Coatings, vol. 7, p. 128, 2017.

39. Heryanto, B. Abdullah, D. Tahir, and Mahdalia, "Quantitative analysis of X-Ray diffraction spectra for determine structural properties and deformation energy of Al, Cu and Si," Journal of Physics: Conference Series, vol. 1317, p. 012052, 2019.

40. D. D. a. G. Kimmel, G. A. Frank and A. Landau, "X-Ray Diffraction (XRD) Characterization of Microstrain in Some Iron and Uranium Alloys," 1996.

41. A. R. Bushroa, R. G. Rahbari, H. H. Masjuki, and M. R. Muhamad, "Approximation of crystallite size and microstrain via XRD line broadening analysis in TiSiN thin films," Vacuum, vol. 86, pp. 1107-1112, 2012/02/29/ 2012. 
42. S. Balamurugan, A. R. Balu, K. Usharani, M. Suganya, S. Anitha, D. Prabha, et al., "Synthesis of CdO nanopowders by a simple soft chemical method and evaluation of their antimicrobial activities," Pacific Science Review A: Natural Science and Engineering, vol. 18, pp. 228-232, 2016/11/01/ 2016. 